\title{
ON COMPLETING UNIMODULAR POLYNOMIAL VECTORS OF LENGTH THREE
}

\author{
RAVI A. RAO
}

\begin{abstract}
It is shown that if $R$ is a local ring of dimension three, with $\frac{1}{2} \in$ $R$, then a polynomial three vector $\left(v_{0}(X), v_{1}(X), v_{2}(X)\right)$ over $R[X]$ can be completed to an invertible matrix if and only if it is unimodular. In particular, if $1 / 3 ! \in R$, then every stably free projective $R\left[X_{1}, \ldots, X_{n}\right]$-module is free.
\end{abstract}

\section{INTRODUCTION}

In [6] A. Suslin queries

A. Suslin's question $\left(S_{r}(R)\right)$. Let $R$ be a local ring. If $1 / r ! \in R$, can every unimodular $(r+1)$-vector over $R[X]$ be completed to an invertible matrix?

In this note we settle $S_{r}(R)$ when $R$ is a noetherian local ring of Krull dimension three.

Let us briefly recapitulate known results on $S_{r}(R)$. Let $R$ be a two dimensional noetherian local ring. A beautiful theorem of $L$. N. Vaserstein in [8] identifies the set $\mathrm{Um}_{3}(R[X]) / E_{3}(R[X])$ with the Elementary Symplectic Witt group $\mathrm{W}_{\mathrm{E}}(R[X])$. If $1 / 2 \in R$, a well-known theorem of $\mathrm{M}$. Karoubi asserts that any invertible alternating matrix over a polynomial ring $R[X]$ is stably congruent to its constant form. In particular, the Symplectic Witt group $\mathrm{W}(R[X]) \equiv 0$. M. P. Murthy had remarked that these two facts could be used to prove that every $v \in \mathrm{Um}_{3}(R[X])$ can be completed to an invertible matrix. We expanded on this theme of M. P. Murthy, in [3], to show that $S_{d}(R)$ holds. Here we extend the methods in [3] to prove

Theorem. Let $R$ be a noetherian, local ring of Krull dimension three with $1 / 2 \epsilon$ $R$. Then every unimodular 3-vector over $R[X]$ can be completed to an invertible matrix.

The reader can also find some very interesting results on A. Suslin's question, due to M. Roitman, in positive prime characteristics in [5]. The present approach had its genesis in [2], (of course, with roots in Vaserstein theory developed in [8], and guided by M. P. Murthy's remark), where I could extend some of M. Roitman's results in dimensions $\leq 4$.

Received by the editors September 30, 1988 and, in revised form, April 17, 1989.

1980 Mathematics Subject Classification (1985 Revision). Primary 13C10. 


\section{PRELIMINARY REMARKS AND CALCUlations}

All rings $A$ considered in this article will be commutative with an identity element and noetherian. A vector $v=\left(v_{0}, v_{1}, \ldots, v_{r}\right) \in A^{r+1}$ is said to be unimodular if there is a vector $w=\left(w_{0}, w_{1}, \ldots, w_{r}\right) \in A^{r+1}$ such that $v_{0} w_{0}+$ $\cdots+v_{r} w_{r}=1 . \operatorname{Um}_{r+1}(A)$ will denote the set of all unimodular vectors $v \in$ $A^{r+1}$. The group $G l_{r+1}(A)$ of invertible matrices acts on $A^{r+1}$ in a natural way: if $v \in A^{r+1}, \sigma \in G l_{r+1}(A)$ then $\sigma$ will map $v$ to $v \sigma$. Under this action $\mathrm{Um}_{r+1}(A)$ is mapped onto itself; and so $G l_{r+1}(A)$ acts on $\operatorname{Um}_{r+1}(A)$. We let $\sim$ denote equivalence of two vectors under this action. Let $E_{r+1}(A)$ denote the subgroup of $G l_{r+1}(A)$ consisting of all the elementary matrices, i.e. those matrices which are a finite product of matrices of the form $E_{i j}(\lambda), i \neq j$, $\lambda \in A$, which has all its diagonal entries one, has one off-diagonal entry in the $(i, j)$ th position equal $\lambda$, and has all other entries zero. $v \underset{E}{\sim} w$ will denote that $v$ can be elementarily transformed to $w$. Let $\operatorname{Um}_{r+1}(A) / E_{r+1}(A)$ be the set of equivalence classes of vectors $v$ under the equivalence $\tilde{E}_{E}$ induce by the action of $E_{r+1}(A)$ on $\mathrm{Um}_{r+1}(A)$; and let [ $v$ ] denote the equivalence class of $v \in \operatorname{Um}_{r+1}(A)$ in $\operatorname{Um}_{r+1}(A) / E_{r+1}(A)$.

(2.1) W. Van der Kallen's group structure on $\mathrm{Um}_{d+1}(A) / E_{d+1}(A)$. If $A$ is a ring whose maximal spectrum $\operatorname{Max}(A)$ is a finite union of subsets $V_{i}$ where each $V_{i}$, when endowed with the (topology induced from the) Zariski topology is a space of Krull dimension $\leq d$ we shall say that $A$ is essentially of dimension $d$. For instance, a ring of Krull dimension $d$ is obviously essentially of dimension $\leq d$; a local ring of dimension $d$ is essentially of dimension 0 ; whereas a polynomial extension $R[X]$ of a local ring $R$ of dimension $d \geq 1$ has dimension $d+1$ but is essentially of dimension $d$ as $\operatorname{Max}(R[X])=\operatorname{Max}(R /(a)[X]) \cup \operatorname{Max}\left(R_{a}[X]\right)$ for any non-zero-divisor $a \in R$.

In $[9$, Theorem 3.6], W. Van der Kallen has described how one could have an abelian group structure on $\operatorname{Um}_{d+1}(A) / E_{d+1}(A)$. In the sequel we shall always refer to this group structure on $\mathrm{Um}_{d+1}(A) / E_{d+1}(\dot{A})$; and let $*$ denote the group multiplication henceforth. One has

(2.1.1) Remark. Let $A$ be essentially of dimension $d \geq 2$, and let $C_{d+1}(A)$ denote the set of all completable $(d+1)$-vectors in $\operatorname{Um}_{d+1}(A)$. Then,

(i) The map $\sigma \rightarrow\left[e_{1} \sigma\right]$, where $e_{1}=(1,0, \ldots, 0) \in \operatorname{Um}_{d+1}(A)$, is a group homomorphism $S l_{d+1}(A) \rightarrow \operatorname{Um}_{d+1}(A) / E_{d+1}(A)$.

(ii) $C_{d+1}(A) / E_{d+1}(A)$ is a subgroup of $\operatorname{Um}_{d+1}(A) / E_{d+1}(A)$.

Proof. (i) follows from [9, Theorem 3.16(iv)]. Since any $v \in C_{d+1}(A)$ can be completed to a matrix of determinant one, $C_{d+1}(A) / E_{d+1}(A)$ is the image of $S l_{d+1}(A)$ under the homomorphism mentioned in (i); whence it is a subgroup of $\mathrm{Um}_{d+1}(A) / E_{d+1}(A)$. 
(2.2) On A. Suslin's procedure for completing $\left(a_{0}, a_{1}, a_{2}^{2}, \ldots, a_{r}^{r}\right)$. In [6, Proposition 1.6] A. Suslin shows that if $\left(a_{0}, a_{1}, \ldots, a_{r}\right) \in \operatorname{Um}_{r+1}(A)$ then $\left(a_{0}, a_{1}\right.$, $\left.a_{2}^{2}, \ldots, a_{r}^{r}\right)$ can be completed. His proof, as observed by M. P. Murthy in [1, Chapter V, Proposition 1.2], actually demonstrates,

(2.2.1) Proposition. Let $\left(a_{0}, a_{1}, \ldots, a_{r}\right) \in \mathrm{Um}_{r-1}(A)$. Suppose that $\left(\bar{a}_{0}, \bar{a}_{1}\right.$, $\left.\ldots, \bar{a}_{r+1}\right)$ is completable in $\bar{A}=A /\left(a_{r}\right)$. Then $\left(a_{0}, a_{1}, \ldots, a_{r}^{r}\right)$ is completable.

As an application of this proposition we have

(2.2.2) Proposition. Let $R$ be a local ring of dimension 3 with $1 / 2 \in R$. Let $v=\left(v_{0}, v_{1}, v_{2}, v_{3}\right) \in \operatorname{Um}_{4}(R[X])$. Then $v$ is completable if and only if $v^{(2)}=\left(v_{0}^{2}, v_{1}, v_{2}, v_{3}\right)$ is completable.

Proof. By [3, Example 1.5.3 and Lemma 1.3.1],

$$
\left[v^{(2)}\right]=[v] *[v]
$$

in $\mathrm{Um}_{4}(R[X]) / E_{4}(R[X])$. By Remark 2.1.1, $v$ is completable implies that $v^{(2)}$ is also completable.

Conversely, let $v^{(2)}$ be completable. By [3, Proposition 1.4.4],

$$
v \underset{E}{\sim}\left(w_{0}, w_{1}, w_{2}, c\right)
$$

with $c \in R$ a non-zero-divisor. As mentioned in the introduction (or cf. [3, Theorem 2.5]), since $\operatorname{dim} R /(c)=2$ and $1 / 2 \in R$,

$$
\left(\bar{w}_{0}, \bar{w}_{1}, \bar{w}_{2}\right) \in e_{1} S l_{3}(R /(c)[X]) .
$$

By Proposition 2.2.1, $\left(w_{0}, w_{1}, w_{2}, c^{3}\right)$ is completable. Thus,

(i) $\left(v_{0}, v_{1}, v_{2}, v_{3}^{3}\right) \underset{E}{\sim}\left(w_{0}, w_{1}, w_{2}, c^{3}\right)$ by [10, Theorem],

(ii) $[v]^{n}=\left[\left(v_{0}, v_{1}, v_{2}, v_{3}^{n}\right)\right]$ for all $n$ by [3, Example 1.5.3 and Lemma 1.3.1].

Hence $[v]^{2}=\left[v^{(2)}\right] \in C_{4}(R[X]) / E_{4}(R[X])$, and $[v]^{3}=\left[\left(w_{0}, w_{1}, w_{2}, c^{3}\right)\right] \in$ $C_{4}(R[X]) / E_{4}(R[X])$. By Remark 2.1.1, $[v] \in C_{4}(R[X]) / E_{4}(R[X])$, i.e. $v$ is completable.

(2.3) The elementary symplectic Witt group $\mathrm{W}_{\mathrm{E}}(A)$. If $\alpha \in M_{r}(A), \beta \in M_{s}(A)$ are matrices then $\alpha \perp \beta$ denotes the matrix $\left(\begin{array}{cc}\alpha & 0 \\ 0 & \beta\end{array}\right) \in M_{r+s}(A) . \psi_{1}$ will denote $\left(\begin{array}{cc}0 & 1 \\ -1 & 0\end{array}\right) \in E_{2}(\mathbf{Z})$, and $\psi_{r}$ is inductively defined by $\psi_{r}=\psi_{r-1} \perp \psi_{1} \in E_{2 r}(\mathbf{Z})$, for $r \geq 2$.

A skew-symmetric matrix whose diagonal elements are zero is called an alternating matrix. If $\varphi \in M_{2 r}(A)$ is alternating then $\operatorname{det}(\varphi)=(\operatorname{pf}(\varphi))^{2}$ where pf is a polynomial (called the Pfaffian) in the matrix elements with coefficients \pm 1 . Note that we need to fix a sign in the choice of pf; so insist $\operatorname{pf}\left(\psi_{r}\right)=1$ for all $r$. For any $\alpha \in M_{2 r}(A)$ and any alternating matrix $\varphi \in M_{2 r}(A)$ we have $\operatorname{pf}\left(\alpha^{t} \varphi \alpha\right)=\operatorname{pf}(\varphi) \operatorname{det}(\alpha)$. For alternating matrices $\varphi, \psi$ it is easy to check that $\operatorname{pf}(\varphi \perp \psi)=(\operatorname{pf}(\varphi))(\operatorname{pf}(\psi))$. 
Two matrices $\alpha \in M_{r}(A), \beta \in M_{s}(A)$ are said to be equivalent (w.r.t. $E A$ ) if there is a $\varepsilon \in E_{2(r+s+l)}(A)$, for some $l$, such that $\alpha \perp \psi_{s+l}=\varepsilon^{\mathrm{t}}\left(\beta \perp \psi_{s+l}\right) \varepsilon$, (the $\mathrm{t}$ stands for 'transpose'). Denote this by $\alpha \underset{E}{\beta} \beta \underbrace{\sim}_{E}$ is an equivalence relation; denote by $[\alpha]$ the orbit of $\alpha$ under this relation. Moreover, a matrix equivalent to an alternating matrix is itself alternating and has the same Pfaffian.

It is easy to see (cf. [8, p. 945]) that $\perp$ induces the structure of an abelian group on the set of all equivalence classes of alternating matrices with Pfaffian 1 ; this group is called the Elementary Symplectic Witt group and is denoted by $\mathrm{W}_{\mathrm{E}} A$.

(2.4) M. Karoubi's theorem and square roots in $\mathrm{W}_{\mathrm{E}}(R[X])$. A famous theorem of M. Karoubi asserts that any invertible alternating matrix $V(X)$ over a polynomial ring $R[X]$ is stably congruent to its constant form if $1 / 2 \in R$, i.e. there is an $l$, and a $\sigma \in S l_{s}(R[X])$, for suitable $s$, such that $\sigma^{\mathrm{t}}\left(V(X) \perp \psi_{l}\right) \sigma=$ $V(0) \perp \psi_{l}$. The machination of M. Karoubi's proof (cf. [8, §3]) gives

(2.4.1) Proposition. Let $R$ be a local ring with $1 / 2 k \in R$, and let $[V] \in$ $\mathrm{W}_{\mathrm{E}}(R[X])$. Then $[V]$ has a kth root, i.e. there is a $[W] \in \mathrm{W}_{\mathrm{E}}(R[X])$ such that $[V]=[W]^{k}$ in $\mathrm{W}_{\mathrm{E}}(R[X])$.

Proof. Since $R$ is local $\mathrm{W}_{\mathrm{E}}(R) \equiv 0$, so we may assume that $V(0)=\psi_{r}$ for some $r$. Let me describe M. Karoubi's process showing $V$ is stably congruent to $V(0)$; for details consult $[8, \S 3]$. The first step is to "stably make $V(X)$ linear" (known as the "Higman trick")-i.e. find an $\varepsilon \in E_{2(r+t)}(R[X])$ such that

$$
\varepsilon^{\mathrm{t}}\left(V \perp \psi_{t}\right)=\psi_{r+t}+n X,
$$

for some $t \geq 0$, some $n \in M_{2(r+t)}(R)$.

Since $\gamma=I_{r+t}-\psi_{r+t} n X \in S l_{2(r+t)}(R[X]), \psi_{r+t} n$ is nilpotent, i.e. $\left(\psi_{r+t} n\right)^{l} \equiv 0$ for some $l$. Hence, if $1 / 2 k \in R$, we can extract a $k$ th root of $\gamma \quad\left(=\beta^{2 k}\right.$ say) for some $\beta \in S l_{2(r+t)}(R[X])$. Now M. Karoubi pointed out that

$$
\varepsilon^{\mathrm{t}}\left(V \perp \psi_{t}\right) \varepsilon=\psi_{r+t} \gamma=\psi_{r+t} \beta^{2 k}=\left(\beta^{k}\right)^{\mathrm{t}} \psi_{r+t} \beta^{k} .
$$

Let $W=\beta^{\mathrm{t}} \psi_{r+t} \beta$. Then applying Whitehead's lemma one can check that $W \perp W \perp \cdots \perp W(k$ times $) \underset{E}{\sim} V$, i.e. $[V]=[W]^{k}$ in $\mathrm{W}_{\mathrm{E}}(R[X])$.

(2.5) The antipodal vectors equality in $\mathrm{Um}_{3}(R[X])$ in small dimensions. In [3, Lemma 1.3.1] we showed that if a $v=\left(v_{0}, v_{1}, \ldots, v_{d}\right) \in \operatorname{Um}_{d+1}(A)$, where $A$ is essentially of dimension $d$, can be elementarily transformed to (its antipodal vector) $-v=\left(-v_{0}, v_{1}, \ldots,-v_{d}\right)$ then for all $n,\left[\left(v_{0}^{n}, v_{1}, \ldots, v_{d}\right)\right]=[v]^{n}$ in $\mathrm{Um}_{d+1}(A) / E_{d+1}(A)$. There are many examples of vectors which cannot be elementarily transformed to their antipodal vector; but in $[3, \S 1.5]$ we showed that if $A=R[X], R$ a local ring of dimension 2 with $1 / 2 \in R$, then for any $v \in \mathrm{Um}_{3}(R[X]), v \underset{E}{\sim}-v$. Here, by a different argument, we show that 
(2.5.1) Proposition. Let $R$ be a local ring of dimension $\leq 4$ with $1 / 2 \in R$ and let $v=\left(v_{0}, v_{1}, v_{2}\right) \in \operatorname{Um}_{3}(R[X])$. Then $v=\left(v_{0}, v_{1}, v_{2}\right) \underset{E}{\sim}\left(-v_{0},-v_{1},-v_{2}\right)$ $=-v$.

Proof. Choose a $w=\left(w_{0}, w_{1}, w_{2}\right)$ such that $v_{0} w_{0}+v_{1} w_{2}+v_{2} w_{2}=1$, and consider the alternating matrix $V$ with Pfaffian 1 given by

$$
V(v, w)=\left\{\begin{array}{cccc}
0 & v_{0} & v_{1} & v_{2} \\
-v_{0} & 0 & w_{2} & -w_{1} \\
-v_{1} & -w_{2} & 0 & w_{0} \\
-v_{2} & w_{1} & -w_{0} & 0
\end{array}\right\} \in S l_{4}(R[X])
$$

Since $1 / 2 \in R$, by M. Karoubi's theorem (cf. $\S 2.4$ ) there is a

$$
\beta \in S l_{4+2 l}(R[X]),
$$

for some $l$, such that $\beta^{\mathrm{t}}\left(V \perp \psi_{l}\right) \beta=\psi_{l+2}$. Since $\operatorname{dim} R \leq 4$, by [7, Theorem 2.6], $\mathrm{Um}_{r}(R[X])=e_{1} E_{r}(R[X])$ for all $r \geq 6$. Hence on applying [8, Lemma 5.5 and Lemma 5.6] we can find a $\beta^{*} \in S l_{4}(R[X])$ such that $\left(\beta^{*}\right)^{t} V \beta^{*}=\psi_{2}$.

Let $\delta=$ diagonal $(-1,1,-1,1) \in E_{4}(R)$. Then $\delta^{\mathrm{t}} \psi_{2} \delta=-\psi_{2}$. Thus

$$
\delta^{\mathrm{t}}\left(\beta^{*}\right)^{\mathrm{t}} V \beta^{*} \delta=\delta^{\mathrm{t}} \psi_{2} \delta=-\psi_{2}=\psi_{2}^{\mathrm{t}}=\left[\left(\beta^{*}\right)^{\mathrm{t}} V \beta^{*}\right]^{\mathrm{t}}=\left(\beta^{*}\right)^{\mathrm{t}} V^{\mathrm{t}} \beta^{*},
$$
and so if $\sigma=\left(\beta^{*}\right)^{\mathrm{t}}$ then $\left(\sigma^{-1} \delta^{\mathrm{t}} \sigma\right) V\left(\sigma^{-1} \delta^{\mathrm{t}} \sigma\right)^{\mathrm{t}}=-V$.

By [7, Corollary 1.4] $\sigma^{-1} \delta^{t} \sigma \in E_{4}(R[X])$. Now the equation (*) will prove the proposition on applying [11, Theorem 10].

(2.5.2) Remark. The above argument can be suitably modified to show that if $[V] \in \mathrm{W}_{\mathrm{E}}(R[X])$, where $R$ is a local ring with $1 / 2 \in R$, then $[V]=[-V]$ in $\mathrm{W}_{\mathrm{E}}(R[X])$.

(2.6) "Coordinate squares" in $\mathrm{W}_{\mathrm{E}}(R[X])$. Let us say that an invertible alternating matrix $V$ is a "coordinate $k$ th power" if the first row of $V$ has the form $\left(0, v_{1}^{k}, v_{2}, \ldots, v_{2 r-1}\right)$. It would be of interest to know if, under congenial conditions, the above fact, proven in Proposition 2.4.1, that every $[V] \in \mathrm{W}_{\mathrm{E}}(R[X])$ is a $k$ th power in $\mathrm{W}_{\mathrm{E}}(R[X])$ (under suitable hypothesis on $R$ ) can be translated to read that $[V]$ has a representative $V^{*}$ which is a coordinate $k$ th power and which, moreover, has the same size as that of $V$. We give some evidence for this here.

Firstly recall some multiplicative relations in $\mathrm{W}_{\mathrm{E}}(A)$ observed by $\mathrm{L}$. N. Vaserstein in [8, Theorem 5.2( $\left.\left.\mathrm{a}_{2}\right)\right]$.

(2.6.1) The Vaserstein Rule. Let $v_{1}=\left(a_{0}, a_{1}, a_{2}\right), v_{2}=\left(a_{0}, b_{1}, b_{2}\right)$ be unimodular vectors. Suppose that $a_{0} a_{0}^{\prime}+a_{1} a_{1}^{\prime}+a_{2} a_{2}^{\prime}=1$, and that

$$
v_{3}=\left(a_{0},\left(b_{1}, b_{2}\right)\left(\begin{array}{cc}
a_{1} & a_{2} \\
-a_{2}^{\prime} & a_{1}^{\prime}
\end{array}\right)\right) \in \operatorname{Um}_{3}(A) \text {. }
$$

Then for any $w_{1}, w_{2}, w_{3}$ such that $v_{i} \cdot w_{i}^{\mathrm{t}}=1, i=1,2,3$, we have

$$
\left[V\left(v_{1}, w_{1}\right)\right] \perp\left[V\left(v_{2}, w_{2}\right)\right]=\left[V\left(v_{3}, w_{3}\right)\right] \text { in } \mathbf{W}_{\mathrm{E}}(A) \text {. }
$$

(Note. $V(v, w)$ is defined in Proposition 2.5.1, and $[V(v, w)]$ is well defined in $\mathrm{W}_{\mathrm{E}}(A)$ via $[8$, Lemma 5.1].) 
(2.6.2) Corollary. (i) Let $v_{1}=\left(a_{0}, a_{1}, a_{2}\right), v_{2}=\left(b_{0}, a_{1}, a_{2}\right)$ be unimodular vectors. Suppose that $a_{0} a_{0}^{\prime}+a_{1} a_{1}^{\prime}+a_{2} a_{2}^{\prime}=1$ and that $v_{3}=\left(a_{0}\left(b_{0}+a_{0}^{\prime}\right)-1\right.$, $\left.\left(b_{0}+a_{0}^{\prime}\right) a_{1}, a_{2}\right) \in \operatorname{Um}_{3}(A)$. Then for any $w_{1}, w_{2}, w_{3}$ such that $v_{1} \cdot w_{i}^{t}=1$, $i=1,2,3$, we have

$$
\left[V\left(v_{1}, w_{1}\right)\right] \perp\left[V\left(v_{2}, w_{2}\right)\right]=\left[V\left(v_{3}, w_{3}\right)\right] \text { in } \mathbf{W}_{\mathbf{E}}(A) .
$$

(ii) Let $v_{1}=\left(a_{0}, a_{1}, a_{2}\right), v_{2}=\left(b_{0}^{2}, a_{1}, a_{2}\right)$ be unimodular vectors. Suppose that $v_{3}=\left(a_{0} b_{0}^{2}, a_{1}, a_{2}\right)$ and that $w_{1}, w_{2}, w_{3}$ are such that $v_{i} w_{i}^{\mathrm{t}}=1, i=$ $1,2,3$, then

$$
\left[V\left(v_{1}, w_{1}\right)\right] \perp\left[V\left(v_{2}, w_{2}\right)\right]=\left[V\left(v_{3}, w_{3}\right)\right] \text { in } \mathrm{W}_{\mathrm{E}}(A) .
$$

Proof. (i) is immediate from the Vaserstein Rule. We refer the reader to [9, Theorem 3.16(iii)] for deriving (ii) from (i). Note: You may need the Roitman lemma in [5, Lemma 1].

(2.6.3) The "antipodal vectors equality" lemma in $\mathrm{W}_{\mathrm{E}}(A)$. Let $v=\left(v_{0}, v_{1}, v_{2}\right)$ be a unimodular vector and assume that $v \underset{E}{\sim}-v_{E}^{\sim}\left(-v_{0},-v_{1},-v_{2}\right)$. Let $v_{1}^{(2)}=\left(v_{0}^{2}, v_{1}, v_{2}\right)$ and let $w, w_{1}$ be such that $v \cdot w^{\mathrm{t}}=v^{(2)} \cdot w_{1}^{\mathrm{t}}=1$. Then

$$
[V(v, w)]^{2}=\left[V\left(v^{(2)}, w_{1}\right)\right] \text { in } \mathrm{W}_{\mathrm{E}}(A) .
$$

Proof. Imitate the argument in [3, Lemma 1.3.1] in $\mathrm{W}_{\mathrm{E}}(A)$. (Note. You will need Corollary 2.6.2(ii) above.)

Finally, we give some conditions under which we can extract "coordinate squares" in $\mathrm{W}_{\mathrm{E}}(R[X])$;

(2.6.4) Corollary. Let $R$ be a local ring of dimension $\leq 4$ with $1 / 2 \in R$ and let $v=\left(v_{0}, v_{1}, v_{2}\right), v^{(2)}=\left(v_{0}^{2}, v_{1}, v_{2}\right)$ be unimodular $R[X]$-vectors. Let $w, w_{1}$ such that $v \cdot w^{\mathrm{t}}=v^{(2)} \cdot w_{1}^{\mathrm{t}}=1$. Then,

$$
[V(v, w)]^{2}=\left[V\left(v^{(2)}, w_{1}\right)\right] \text { in } \mathrm{W}_{\mathrm{E}}(R[X]) .
$$

Proof. This will follow from Proposition 2.5.1 and Lemma 2.6.3.

(2.6.5) Proposition. Let $R$ be a local ring of dimension $\leq 3$ with $1 / 2 \leq R$ and let $V \in S l_{4}(R[X])$ be an alternating matrix with Pfaffian 1 . Then $[V]=\left[V^{*}\right]$ in $\mathrm{W}_{\mathrm{E}}(R[X])$ with $V^{*} \in S l_{4}(R[X])$ a coordinate square. Consequently, there is a stably elementary $\gamma \in S l_{4}(R[X])$ such that $V=\gamma^{\mathrm{t}} V^{*} \gamma$.

Proof. By Proposition 2.4.1, $[V]=[W]^{2}$ for some $[W] \in \mathrm{W}_{\mathrm{E}}(R[X])$. By [7, Theorem 2.6] $\mathrm{Um}_{r}(R[X])=e_{1} E_{r}(R[X])$ for all $r \geq 5$, and so on applying [8, Lemma 5.3 and Lemma 5.5] a few times, if necessary, we can find an alternating matrix $W^{*} \in S l_{r}(R[X])$ (with Pfaffian 1) such that $[W]=\left[W^{*}\right]$. Now apply Corollary 2.6.4 to find $V^{*}$ as required. The last statement follows as above (only applying [8, Lemma 5.5 and Lemma 5.6] instead). 


\section{THE MAIN THEOREM}

(3.1) Theorem. Let $R$ be a local ring of Krull dimension three with $1 / 2 \in R$ and let $v=\left(v_{0}, v_{1}, v_{2}\right)$ be a unimodular 3-vector over $R[X]$. Then $v$ can be completed to an invertible matrix.

Proof. Choose a $w=\left(w_{0}, w_{1}, w_{2}\right)$ such that $v_{0} w_{0}+v_{1} w_{1}+v_{2} w_{2}=1$, and consider the alternating matrix $V$ with Pfaffian 1 given by

$$
V=\left\{\begin{array}{cccc}
0 & v_{0} & v_{1} & v_{2} \\
-v_{0} & 0 & w_{2} & -w_{1} \\
-v_{1} & -w_{2} & 0 & w_{0} \\
-v_{2} & w_{1} & -w_{0} & 0
\end{array}\right\} \in S l_{4}(R[X])
$$

Since $1 / 2 \in R$, by M. Karoubi's theorem (see (*) in Proposition 2.4.1) there is a $\alpha \in S l_{4+l}(R[X])$, for some $l$, such that $\alpha^{\mathrm{t}}\left(V \perp \psi_{l}\right) \alpha=\psi_{l+2}$.

Since $\operatorname{dim} R=3$, by [7, Theorem 2.6] $\operatorname{Um}_{r}(R[X])=e_{1} E_{r}(R[X])$ for all $r \geq 6$. Hence on applying [8, Lemma 5.5 and Lemma 5.6] we can find an $\alpha \in S l_{4}(R[X])$ such that $\alpha^{\mathrm{t}} V \alpha=\psi_{2}$. Consider $e_{4} \alpha^{\mathrm{t}}$, where $e_{4}=(0,0,0,1)$.

By [3, Proposition 1.4.4] $e_{4} \alpha^{\mathrm{t}} \tilde{E}\left(a_{0}(X), a_{1}(X), a_{2}(X), c\right)$, where $c \in R$ is a non-zero-divisor in $R$. Let the 'overbar' denote 'modulo (c)'. By [3, Proposition 2.2], $\left(\overline{a_{0}(X)}, \overline{a_{1}(X)}, \overline{a_{2}(X)}\right) \underset{E}{\sim}\left({\overline{b_{0}(X)}}^{2}, \bar{b}_{1}(X), b_{2}(X)\right)$, for some $b_{0}(X), b_{1}(X), b_{2}(X) \in R[X]$. On "lifting" this elementary map, and after an appropriate elementary transformation further, we can arrange that $e_{4} \alpha^{\mathrm{t}} \tilde{E}$ $\left(b_{0}(X)^{2}, b_{1}(X), b_{2}(X), c\right)$.

By Proposition 2.2.2, $\left(b_{0}(X), b_{1}(X), b_{2}(X), c\right)$ can be completed to an invertible matrix, say $\beta \in S l_{4}(R[X])$ with $e_{4} \beta=\left(b_{0}(X), b_{1}(X), b_{2}(X), c\right)$.

Via Remark 1.1.1 follows that

$$
\begin{aligned}
e_{4} \beta^{-2} \alpha^{\mathrm{t}} & =\left[e_{4} \beta^{-2}\right] *\left[e_{4} \alpha^{\mathrm{t}}\right]=\left[e_{4} \beta\right]^{-2} *\left[e_{4} \alpha^{\mathrm{t}}\right] \\
& =\left(\left[\left(b_{0}(X), b_{1}(X), b_{2}(X), c\right)\right]^{2}\right)^{-1} *\left[e_{4} \alpha^{\mathrm{t}}\right]=\left[e_{4} \alpha^{\mathrm{t}}\right]^{-1} *\left[e_{4} \alpha^{\mathrm{t}}\right] \equiv 1,
\end{aligned}
$$

the last equality being deduced via [3, Example 1.5.3 and Lemma 1.3.1]. Thus, $\beta^{-2} \alpha^{t}=\varepsilon^{\prime} \delta^{\prime}$ for some $\varepsilon^{\prime} \in E_{4}(R[X])$ and $\delta^{\prime}=\left(\begin{array}{ll}1 & 0 \\ 0 & \delta\end{array}\right)$ with $\delta \in S l_{3}(R[X])$.

Now $\psi_{2}=\alpha^{\mathrm{t}} V \alpha=\left(\beta^{2} \varepsilon^{\prime} \delta^{\prime}\right) V\left(\beta^{2} \varepsilon^{\prime} \delta^{\prime}\right)^{\mathrm{t}}=\beta^{2} V^{*}\left(\beta^{2}\right)^{\mathrm{t}}$, where $e_{1} V^{*}=\left(0, v \delta^{\mathrm{t}} \varepsilon\right)$ for some $\varepsilon \in E_{3}(R[X])$-this will follow as $\delta^{\prime}=\left(\begin{array}{ll}1 & 0 \\ 0 & \delta\end{array}\right)$ and via [11, Theorem 10].

By Proposition 2.6.5 there is a stably elementary $\gamma \in S l_{4}(R[X])$ such that $\beta V^{*} \beta^{\mathrm{t}}=\gamma^{\mathrm{t}} V^{* *} \gamma$, with $V^{* *} \in S l_{4}(R[X])$ a coordinate square. Let $e_{1} V^{* *}=$ $\left(0, a^{2}, b, c\right)$, and let $\alpha_{0}$ (cf. $\left.\S 2.2\right)$ be a completion of $\left(a^{2}, b, c\right)$.

Since

it follows via [8, Lemma 5.1] that

$$
c_{1} V^{* *}=e_{1}\left(\begin{array}{cc}
1 & 0 \\
0 & \alpha_{0}
\end{array}\right)^{\mathfrak{t}} \psi_{2}\left(\begin{array}{cc}
1 & 0 \\
0 & \alpha_{0}
\end{array}\right)
$$

$$
V^{* *}=\varepsilon_{1}^{\mathrm{t}}\left(\begin{array}{cc}
1 & 0 \\
0 & \alpha_{0}
\end{array}\right)^{\mathrm{t}} \psi_{2}\left(\begin{array}{cc}
1 & 0 \\
0 & \alpha_{0}
\end{array}\right) \varepsilon_{1}
$$


for some $\varepsilon_{1} \in E_{4}(R[X])$. Thus,

$$
\beta V^{*} \beta^{\mathrm{t}}=\gamma^{\mathrm{t}} V^{* *} \gamma=\gamma^{\mathrm{t}} \varepsilon_{1}^{\mathrm{t}}\left(\begin{array}{cc}
1 & 0 \\
0 & \alpha_{0}
\end{array}\right)^{\mathrm{t}} \psi_{2}\left(\begin{array}{cc}
1 & 0 \\
0 & \alpha_{0}
\end{array}\right) \varepsilon_{1} \gamma
$$

Hence,

$$
\begin{array}{r}
\beta^{-1}\left[\left(\begin{array}{cc}
1 & 0 \\
0 & \alpha_{0}^{-1}
\end{array}\right)^{\mathrm{t}}\left(\varepsilon_{1}^{-1}\right)^{\mathrm{t}}\left(\gamma^{-1}\right)^{\mathrm{t}}\right] \beta V^{*} \beta^{\mathrm{t}}\left[\gamma^{-1} \varepsilon_{1}^{-1}\left(\begin{array}{cc}
1 & 0 \\
0 & \alpha_{0}^{-1}
\end{array}\right)\right]\left(\beta^{-1}\right)^{\mathrm{t}} \\
=\beta^{-1} \psi_{2}\left(\beta^{-1}\right)^{\mathrm{t}}=\beta^{-1}\left(\beta^{2} V^{*}\left(\beta^{2}\right)^{\mathrm{t}}\right)\left(\beta^{-1}\right)^{\mathrm{t}}=\beta V^{*} \beta^{\mathrm{t}}=\gamma^{\mathrm{t}} V^{* *} \gamma ;
\end{array}
$$

and so if

$$
\theta=\beta^{\mathrm{t}} \gamma^{-1} \varepsilon_{1}^{-1}\left(\begin{array}{cc}
1 & 0 \\
0 & \alpha_{1}^{-1}
\end{array}\right)\left(\beta^{\mathrm{t}}\right)^{-1} \gamma^{-1}, \text { then } \theta^{\mathrm{t}} V^{*} \theta=V^{* *} .
$$

Compute $e_{4} \theta^{\mathrm{t}}$ in the abelian group $\operatorname{Um}_{4}(R[X]) / E_{4}(R[X])$ via Remark 2.1.1 to get $\left[e_{4} \theta^{\mathrm{t}}\right]=\left[e_{4}\left(\gamma^{\mathrm{t}}\right)^{-1}\right]^{2}$. But $\gamma$ is stably elementary and so via [3, Proposition 2.6] $\left[e_{4}\left(\gamma^{\mathrm{t}}\right)^{-1}\right]^{2}=1$; hence $\left[e_{4} \theta^{\mathrm{t}}\right]=1$, i.e. $e_{4} \theta^{\mathrm{t}} \underset{E}{\sim} e_{4}$. Hence

$$
\theta^{\mathrm{t}} \varepsilon^{\prime}=\left(\begin{array}{cc}
1 & 0 \\
0 & \left(\theta^{\prime}\right)^{\mathfrak{t}}
\end{array}\right)
$$

for some $\theta^{\prime} \in S l_{3}(R[X]), \varepsilon^{\prime} \in E_{4}(R[X])$.

Now

$$
\theta^{\mathrm{t}} V^{*} \theta=\left(\begin{array}{cc}
1 & 0 \\
0 & \left(\theta^{\prime}\right)^{\mathrm{t}}
\end{array}\right)\left(\varepsilon^{\prime}\right)^{-1} V^{*}\left(\left(\varepsilon^{\prime}\right)^{-1}\right)^{\mathrm{t}}\left(\begin{array}{cc}
1 & 0 \\
0 & \theta^{\prime}
\end{array}\right)=V^{* *},
$$

and so via [11, Theorem 10] we can deduce that there is an $\varepsilon^{\prime \prime} \in E_{3}(R[X])$ such that $v \varepsilon^{\prime \prime} \theta^{\prime}=\left(a^{2}, b, c\right)$. Since $\left(a^{2}, b, c\right)$ is completable, so is $v$.

Remark. Let us, following M. Krusemeyer, say that a vector $v \in \operatorname{Um}_{r}(A)$ is skew-completable if there is an invertible alternating matrix $V \in S l_{r+1}(A)$ with its first row $e_{1} V=(0, v)$.

By making some appropriate modifications in the argument used to prove Theorem 3.1 one can show that,

(3.2) Theorem. Let $R$ be a local ring of Krull dimension $d$ with $1 / 2 \in R$, and let $v=\left(v_{0}, v_{1}, \ldots, v_{d-1}\right)$ be a skew-completable vector over $R[X]$. Then $v$ can be completed to an invertible matrix.

Finally, using the well-known "Quillen-Suslin" Monic inversion and LocalGlobal principles, one can derive from $S_{d}(R)$ and Theorem 3.1 that,

(3.3) Corollary. Let $R$ be a noetherian ring of dimension 3 with $1 / 6 \in R$. Then any stably extended projective module over $R\left[X_{1}, \ldots, X_{n}\right]$ is extended.

Note added in proof. The contents (especially the mode of proof of the main result) of this note seems of interest in connection with the following problem:

(i) Let $V: \mathrm{Um}_{3}(A) / E_{3}(A) \rightarrow \mathrm{W}_{\mathrm{E}}(A)$ be the Vaserstein symbol. Is this map injective if $\operatorname{dim} A=3$ ? 
I also hope that, after incorporation of some additional theories, the techniques used here will provide some insight towards settling,

(a) Let $R$ be a local ring with $\frac{1}{2} \in R$. Is every $v \in \operatorname{Um}_{3}(R[X])$ completable?

(b) Let $A$ be a smooth affine algebra over the field $\mathbf{C}$ of complex numbers of dimension $d$. Is a stably free A-module of rank $(d-1)$ a free module?

In an article entitled On some actions of stably elementary matrices on alternating matrices we prove that

"Let $A$ have Krull dimension $\leq 5$, and let $V \in S_{4}(A) \cap E_{5}(A)$ be a stably elementary alternating matrix of Pfaffian one. Then $V^{8} \in E_{4}(A)$."

Note. One needs to show that $V \in E_{4}(A)$ to settle (i) above.

We also give some examples of 3 dimensional affine algebras for which the Vaserstein symbol $V$ is bijective.

\section{REFERENCES}

1. S. K. Gupta and M. P. Murthy, Suslin's work on linear groups over polynomial rings and Serre problem, Indian Statistical Institute, New Delhi.

2. R. A. Rao, Two examples of the Bass-Quillen-Suslin conjectures, Math. Ann. 279 (1987), 227-238.

3. tion of A. Suslin, Invent. Math. 93 (1988), 609-618.

4. M. Roitman, On unimodular rows, Proc. Amer. Math. Soc. 95 (1985), 184-188.

5. $\ldots$, On stably extended projective modules over polynomial rings, Proc. Amer. Math. Soc. 97 (1986), 585-589.

6. A. Suslin, On stably free modules, Math. USSR-Sb. 31 (1977), 479-491.

7. _ On the structure of the special linear group over polynomial rings, Math. USSR-Izv. 11 (1977), 221-238.

8. A. Suslin and L. N. Vaserstein, Serre's problem on projective modules over polynomial rings and algebraic $K$-theory, Math. USSR-Izv. 10 (1976), 937-1001.

9. W. Van der Kallen, A group structure on certain orbit sets of unimodular rows, J. Algebra 82 (1983), 363-397.

10. L. N. Vaserstein, Operations on orbits of unimodular vectors, J. Algebra 100 (1986), 456461.

11. _ _ Computation of $K_{1}$ via Mennicke symbols, Comm. Algebra 15 (1987), 611-656.

School of Mathematics, Tata Institute of Fundamental Research, Homi Bhabha Road, BOMBAY 400 005, INDIA 